\title{
Languages with Bounded Multiparty Communication Complexity *
}

\author{
Arkadev Chattopadhyay \\ Andreas Krebs \\ McGill University, Montreal, Canada \\ achatt3ecs.mcgill.ca \\ Universität Tübingen, Germany \\ Michal Koucký \\ mail@krebs-net.de \\ Mario Szegedy \\ Mathematical Institute, Academy of Sciences, Czech Republic \\ Rutgers University, New Jersey, USA \\ koucky@math.cas.cz \\ szegedyecs. rutgers.edu \\ Pascal Tesson \\ Laval University, Québec, Canada \\ Denis Thérien \\ McGill University, Montreal, Canada \\ pascal.tessoneift.ulaval.ca \\ denisecs.mcgill.ca
}

${ }^{*}$ Research supported in part by the NFS (M. Szegedy), NSERC (A. Chattopadhyay, M. Koucký, P. Tesson, D. Thérien), FQRNT (M. Koucký, D. Thérien) and the Alexander von Humboldt Foundation (P. Tesson and D. Thérien). We are also grateful to Pavel Pudlák for suggesting the use of the Hales-Jewett Theorem. 


\begin{abstract}
We study languages with bounded communication complexity in the multiparty "input on the forehead model" with worst-case partition. In the two-party case, languages with bounded complexity are exactly those recognized by programs over commutative monoids [20]. This can be used to show that these languages all lie in shallow $\mathrm{ACC}^{0}$.

In contrast, we use different coding techniques to show that there are languages of arbitrarily large circuit complexity which can be recognized in constant communication by $k$ players for $k \geq 3$. However, if a language has a neutral letter and bounded communication complexity in the $k$-party game for some fixed $k$ then the language is in fact regular and we give an algebraic characterization of regular languages with this property. We also prove that a symmetric language has bounded $k$-party complexity for some fixed $k$ iff it has bounded two party complexity.
\end{abstract}

\title{
1 Introduction
}

The "input on the forehead" multiparty model of communication, introduced by Chandra, Furst and Lipton [7], is a powerful tool in the study of branching programs [2, 6, 7] and shallow-depth Boolean circuits (among many others $[11,14,15]$ ). However, it is still, in many regards, not well-understood as both upper bounds $[1,12]$ and lower bounds $[2,7,19]$ for the model appear very challenging. In particular, good lower bounds on the $k$-party non-interactive communication complexity of an explicit function $f$ when $k>\log n$ have long been sought since they would yield size-lower bounds for $\mathrm{ACC}^{0}$ circuits computing $f$ [9], and even more modest lower bounds $\Omega\left(\log ^{3} n\right)$ for particular functions like Disjointness in three-party setting would imply separation of different proof systems [5].

We obtain significant insight in the multiparty model by focusing on functions that have bounded $k$-party complexity for $k \geq 3$ an arbitrary constant. For the two-party model, languages with bounded communication complexity have many nice characterizations [20] implying, in particular, that any language with bounded two-party complexity can be computed by very shallow $\mathrm{ACC}^{0}$ circuits. In contrast, we show in Section 3 that there are languages with arbitrarily large uniform circuit complexity whose three-party communication complexity is bounded by a constant even for the worst-case partition of the input instances among the players. An analog result for non-uniform circuit complexity can also be derived. These languages are constructed using specially crafted error-correcting codes. Because of these results, we cannot expect to obtain characterizations of languages of bounded multiparty complexity which are as nice as those for the two-player case.

There are several key features that make the multiparty communication model so powerful: first, every input bit is seen by several players, second, every $(k-1)$-tuple of input positions is seen by at least one of the $k$ players, and third, all players know the partitioning of the input, i.e., they know which positions they actually see. Multiparty communication complexity upper bounds typically rely heavily on all these properties. If we remove the first two properties then we obtain essentially the multiparty "input in the hand" model which is computationally even weaker than the two-party communication model. To understand how crucial the last property is, we consider two restricted classes of languages/functions in which this advantage is in some sense taken away. 
First, we consider in Section 4 languages with a neutral letter $[4,3]$, i.e. a letter which can be inserted or deleted at will in an input word. We show that every such language having bounded $k$ party communication complexity for some fixed $k$ is regular. Furthermore, we characterize this class of regular languages in terms of algebraic properties of their minimal automaton. Our results indicate that the presence of a neutral letter is thus a severe handicap in the multiparty game and suggests that it might be easier to prove communication complexity lower bounds under this assumption.

Finally, in Section 5 we use the Ramsey-like theorem of Gallai [10] to prove that for any fixed $k \geq 3$ the symmetric functions that can be computed in bounded $k$-party communication complexity by $k$-players are exactly the symmetric functions that have bounded 2-party complexity.

Two of our main proofs rely on the same lower bound which is of independent interest: In Section 2 we show, using a Ramsey-theoretical argument reminiscent of [7], that $k$ parties need to exchange $\omega(1)$ bits of communication to verify that their $k$ inputs in $\{0,1\}^{n}$ represent a partition of $[n]$.

\section{Multiparty Communication Complexity}

The multiparty model of communication complexity was first introduced by Chandra, Furst and Lipton [7]. In this game, $k$ players $P_{1}, \ldots, P_{k}$ wish to collaborate to compute a function $f: \Sigma^{n} \rightarrow\{0,1\}$. The $n$ input letters are partitioned into $k$ sets $X_{1}, \ldots, X_{k} \subseteq[n]$ and each participant $P_{i}$ knows the values of all the inputs except the ones of $X_{i}$. This game is often referred to as the "input on the forehead" model since it is convenient to picture that player $i$ has the letters of $X_{i}$ written on his forehead, available to everyone but himself. Players exchange bits, according to an agreed upon protocol, by writing them on a public blackboard. The protocol specifies whose turn it is to speak, and what the player broadcasts is a function of the communication history and the input he has access to. The protocol's output is a function of what is on the blackboard after the protocol's termination. We denote by $D_{k}(f)$ the $k$-party communication complexity of $f$, i.e. the minimum number of bits exchanged in a protocol for $f$ on the worst case input and for the worst-case partition of inputs. More generally, we consider functions $f: \Sigma^{*} \rightarrow\{0,1\}$ and thus view $D_{k}(f)$ as a function of input length.

The information available to individual players overlaps a lot since any input letter is known to $k-1$ of the $k$ players. Thus, the power of the multiparty model increases with the number of players involved as the fraction of inputs available to each player increases.

A subset $S$ of $\Sigma^{X_{1} \times \ldots \times X_{k}}$ is a cylinder in the ith dimension if membership in $S$ is independent of the $i$ th coordinate, i.e. if for all $x_{1}, x_{2}, \ldots, x_{k}$ and any $x_{i}^{\prime}$ we have $\left(x_{1}, \ldots, x_{i}, \ldots, x_{k}\right) \in S$ if and only if $\left(x_{1}, \ldots, x_{i}^{\prime}, \ldots, x_{k}\right) \in S$. We say that $S$ is a cylinder intersection if $S=\bigcap_{1 \leq i \leq k} S_{i}$ where $S_{i}$ is a cylinder in the $i$ th dimension. A cylinder intersection is called $f$-monochromatic if the function $f$ evaluates to the same value on every input instance in the intersection. The following lemma underlies all lower bound arguments for the multiparty model:

Lemma 1 (see [14]) Let $f: \Sigma^{X_{1} \times \ldots \times X_{k}} \rightarrow\{0,1\}$ be a function of $k$-inputs. Any $k$-party communication protocol of cost $c$ computing $f$ partitions the input space into at most $2^{c} f$-monochromatic 
cylinder intersections corresponding to the communication exchanged on a particular input.

We say that a set of $k$ elements of $\Sigma^{X_{1} \times \ldots \times X_{k}}$ forms a star if it is of the form:

$$
\left(x_{1}^{\prime}, x_{2}, \ldots, x_{k}\right),\left(x_{1}, x_{2}^{\prime}, \ldots, x_{k}\right), \ldots,\left(x_{1}, x_{2}, \ldots, x_{k}^{\prime}\right)
$$

where the $x_{i}$ are values for the input bits letters in $X_{i}$ for each $i$ with $x_{i} \neq x_{i}^{\prime}$. In that case, we call $\left(x_{1}, x_{2}, \ldots, x_{k}\right)$ the center of this star. These notions lead to a useful characterization of cylinder intersections.

Lemma 2 A set $S \subseteq \Sigma^{X_{1} \times \ldots \times X_{k}}$ is a cylinder intersection if and only if the center of any star contained in $S$ is itself an element of $S$.

A $k$-rectangular reduction $r$ from $L \subseteq\{0,1\}^{n \times k}$ to $K \subseteq\{0,1\}^{l(n) \times k}$ is a $k$-tuple of functions $\left(r_{1}, \ldots, r_{k}\right)$ with each $r_{i}:\{0,1\}^{n} \rightarrow\{0,1\}^{l(n)}$ such that $\left(x_{1}, \ldots, x_{k}\right) \in L$ iff $\left(r_{1}\left(x_{1}\right), \ldots, r_{k}\left(x_{k}\right)\right) \in$ $K$. We call $l$ the length of the reduction. The following simple observation shall be useful:

Observation 3 Let $L \subseteq\{0,1\}^{n \times k}$ and $K \subseteq\{0,1\}^{l(n) \times k}$ be languages such that there exists a rectangular reduction from $L$ to $K$ of length $l$. Then, $D_{k}(L)(n) \leq D_{k}(K)(l(n))$.

Lower bounds for the $k$-party communication complexity of the functions $\operatorname{Part}_{k}$ and $G I P_{k, p}$ will be particularly useful. Both functions take as input an $n \times k$ Boolean matrix $A$ and we think of the $i^{\text {th }}$ column of $A$ as representing a subset $x_{i}$ of $[n]=\{1, \ldots n\}$. We define $\operatorname{Part}_{k}(A)=1$ iff each row contains exactly one 1 (i.e. the $x_{i}$ form a partition of $[n]$ ) and $G I P_{k, p}=1$ iff the number of all- 1 rows of $A$ (i.e. the size of the intersection of the $x_{i}$ ) is divisible by $p$. It is clear that for the $k$-party game the worst input partition for $G I P_{k, p}$ and $\operatorname{Part}_{k}$ is the one where player $P_{i}$ holds the bits of column $i$ on his forehead.

Lemma $4([\mathbf{2}, \mathbf{1 3}]) D_{k}\left(G I P_{k, p}\right)=\Omega(n)$ for all constants $k, p \geq 2$.

More precisely, the best known lower bounds for GIP are $\Omega\left(n / 2^{k}\right)[8,19]$ and hold even for $k$ growing as a function of $n$ but we only consider the case where $k$ is constant.

We establish a lower bound on the $k$-party communication complexity of Part $_{k}$ by applying a Ramsey-theoretical result known as the Hales-Jewett Theorem. The $n$-tuples $v^{1}, \ldots, v^{t} \in[t]^{n}$ are said to form a combinatorial line if the $v^{j}$ are distinct and for each $1 \leq i \leq n$ either all the $v^{j}$ agree on position $i$ (i.e. $v_{i}^{j}=v_{i}^{j^{\prime}}$ for all $1 \leq j \leq j^{\prime} \leq t$ ) or we have $v_{i}^{j}=j$ for all $1 \leq j \leq t$.

Theorem 5 (Hales-Jewett [10]) For any integers $c, t$ there exists an integer $n$ such that if all vectors in $[t]^{n}$ are colored with $c$ colors then there is a monochromatic combinatorial line $v^{1}, \ldots, v^{t}$ (i.e. a line whose elements all were assigned the same color).

We now prove: 
Lemma 6 For all $k, D_{k}\left(\right.$ Part $\left._{k}\right)=\omega(1)$.

Proof: Consider the input as a collection of $k$ subsets of $[n]$. Every input $\left(S_{1}, \ldots, S_{k}\right) \in \mathcal{P}([n])^{k}$ that is accepted by a protocol for Part $_{k}$ is such that for every $1 \leq j \leq n$, the element $j$ lies in exactly one of the $S_{i}$. Using this observation, these inputs can be put in one-to-one correspondence with $n$-tuples in $[k]^{n}$. As an example for $k=3$ and $n=4$, we have $\operatorname{Part}_{3}(\{4\},\{1,3\},\{2\})=1$ and this input corresponds to the $n$-tuple $(2,3,2,1)$.

Suppose that the $k$-party communication complexity of Part $_{k}$ is bounded, for some $k$, by a constant $c$. To every input accepted by a protocol for $\operatorname{Part}_{k}$, (i.e. to every element in $[k]^{n}$ ), we assign one of $2^{c}$ colors corresponding to the communication history resulting from that particular input. If $n$ is large enough then by the Hales-Jewett Theorem this set contains a monochromatic combinatorial line $v^{1}, \ldots, v^{k}$. Let $T \subseteq[n]$ be the (non-empty) set of positions on which the $v^{j}$ differ and for each $i \leq k$ denote as $S_{i}$ the set of positions on which all the $v^{j}$ are $i$. By definition of the above one-to-one correspondence, we have that $T, S_{1}, \ldots, S_{k}$ form a partition of $[n]$ and all the inputs $\left(S_{1} \cup T, S_{2}, \ldots S_{k}\right)$, $\left(S_{1}, S_{2} \cup T, \ldots S_{k}\right), \ldots,\left(S_{1}, S_{2}, \ldots S_{k} \cup T\right)$ induce the same communication history. By Lemma 2, and since these inputs form a star, their center $\left(S_{1}, S_{2}, \ldots S_{k}\right)$ also induces that same communication and must thus belong to $\operatorname{Part}_{k}$. However $S_{1} \cup \ldots \cup S_{k}=[n]-T \neq[n]$ so we get a contradiction.

Note that an $n \times k$ matrix $A$ belongs to Part $_{k}$ iff none of its rows contains two 1 and the total number of 1 entries in $A$ is $n$. If $k \geq 3$ then $k$ players can check the first condition using $k$ bits of communication since any pair of input bits is accessible to at least one player. They are then left with verifying that the sum of the input bits is $n$ which can, surprisingly, be achieved with a communication cost much less than the trivial $O(\log n)$ [7].

\section{Functions with bounded multiparty complexity but high time/space complexity}

In this section we exhibit languages of arbitrarily large computational complexity but with bounded multiparty communication complexity. For a language $L$ and an encoding $C:\{0,1\}^{*} \rightarrow\{0,1\}^{*}$, we denote by $C(L)$ the set $\{C(x) ; x \in L\}$. We prove that for a suitably chosen error-correcting code $C$, any language $L$ is such that its encoding $C(L)$ has bounded multiparty communication complexity. We will choose $C$ such that the corresponding encoding and decoding function are efficiently computable and hence the complexities of $L$ and $C(L)$ will be closely related.

As a warm-up we start with the unary encoding $C_{U}$ defined as follows: for $x \in\{0,1\}^{*}, C_{\mathrm{U}}(x)=$ $0^{x} 10^{2^{n}-x-1}$, where $n$ is the length of $x$ and $x$ is interpreted as an integer between 0 and $2^{n}-1$. Hence, $C_{\mathrm{U}}$ encodes bit strings of length $n$ into strings of length $2^{n}$ having a single 1 in a one-to-one way.

Lemma 7 For any language $L$ and integer $k \geq 3, D_{k}\left(C_{\mathrm{U}}(L)\right) \leq 3$. 
Proof: Without loss of generality $k=3$. On an input $w$ that is split among the three parties, the players need to verify two things: 1) whether $w$ is a valid encoding of some string $x$, and 2) whether the corresponding string $x$ is in $L$. To verify the first property the players only need to check whether at least one of them sees a 1 and whether none of them sees two or more 1s. They can communicate their observations regarding this using six bits in total. Next, one of the players who sees the one, determines the unique string $x$ with $C_{\mathrm{U}}(x)=w$. He can do this solely based on the position of the one since he knows how $w$ is partitioned. This player can also determine whether $x \in L$ and hence $w \in C_{\mathrm{U}}(L)$. He communicates his conclusion to the other parties by sending one more bit. Hence in total players exchange at most seven bits. The protocol can be optimized so that each player simultaneously sends one bit of information for the total of three bits.

The disadvantage of the unary encoding is its inefficiency: because codewords are exponentially longer than the words they encode, we cannot provide efficient reductions between $L$ and $C(L)$. A better encoding can be obtained by concatenating Reed-Solomon codes with the unary encoding. In the 3-party scenario at least one of the parties has on its forehead at least a $1 / 3$-fraction of the input. Hence, if the chosen encoding has the property that from an arbitrary $1 / 3$-fraction of the input the whole word can be reconstructed (assuming the input is an encoding of some word, i.e., assuming that the input is a codeword) the other two parties can reconstruct the whole input and verify whether the parts on remaining foreheads are consistent with such an input. With the proper choice of parameters Reed-Solomon codes have this property.

Let $n$ be a large enough integer, $m=\left\lceil\log _{2} 3 n\right\rceil$ and $d=n / m$. Any string $x \in\{0,1\}^{n}$ can be interpreted as a sequence of $d$ elements from $G F\left[2^{m}\right]$. Define $p_{x}$ to be the degree $d-1$ polynomial over $G F\left[2^{m}\right]$ whose coefficients are given by $x$. Define the Reed-Solomon encoding by $C_{\mathrm{RS}}(x)=$ $p_{x}\left(g_{0}\right) p_{x}\left(g_{1}\right) \cdots p_{x}\left(g_{3 d-1}\right)$, where $G F\left[2^{m}\right]=\left\{g_{0}, g_{1}, \ldots, m_{2^{m}-1}\right\}=\{0,1\}^{m}$. Furthermore, define the concatenation of the Reed-Solomon encoding with the unary encoding by $C_{\mathrm{RSoU}}(x)=$ $C_{\mathrm{U}}\left(p_{x}\left(g_{0}\right)\right) \cdots C_{\mathrm{U}}\left(p_{x}\left(g_{3 d-1}\right)\right)$. Codewords thus consist of $3 d$ blocks of $2^{m}$ bits (corresponding to the $3 d$ symbols of the Reed-Solomon encoding) with each block containing exactly one 1 . Thus, $C_{\mathrm{RSoU}}$ encodes strings of length $n$ into strings of length $O\left(n^{2}\right)$. Furthermore, $C_{\mathrm{RSoU}}$ can be encoded and decoded in polynomial time and so the languages $L$ and $C_{\mathrm{RSoU}}(L)$ are polynomial-time equivalent. Note that the decoding task at hand does not require us to perform error correction in the usual sense: we simply want to identify if an input is a codeword (since we reject all words that are not codewords) and we only care about decoding true codewords.

Lemma 8 For any language $L$ and any $k \geq 3, D_{k}\left(C_{\mathrm{RS} \circ \mathrm{U}}(L)\right) \leq 6$

Proof: Without loss of generality $k=3$ as all but the first two players can pretend they are the same party. Let $m=\left\lceil\log _{2} 3 n\right\rceil$ and $d=n / m$. To check if an input is a codeword, the players can easily check that there are never two 1s in a single block of input bits. They cannot, however, verify at constant cost that each of the $3 d$ blocks contains at least one 1 since this task is essentially the partition problem whose complexity we lower bounded in Lemma 6. We proceed differently: an 
input $w$ of length $3 d \cdot 2^{m}$ can only be a codeword if at least one player (say player 1 ) has on its forehead at least $d$ ones and this player can be identified with three bits of communication. These $d$ ones determine $d$ elements of $G F\left[2^{m}\right]$ hence players 2 and 3 can each privately reconstruct from them the unique degree $d-1$ polynomial $p$ that coincides with these elements. Players 2 and 3 now know that if the input is a codeword then it must be the one corresponding to $p$ and player 2 can check that the bits on player 3's forehead are consistent with that hypothesis while player 3 can similarly crosscheck the input bits on player 2's forehead. If this cross-checking procedure is successful, player 2 can determine the unique $x$ such that $p_{x}=p$, verify $x \in L$ and send the result to all parties. Overall, only six bits of communication suffice to decide if the input is from $C_{\mathrm{RS} \circ \mathrm{U}}(L)$.

As an immediate corollary to this lemma and the fact that the complexity of $C_{\mathrm{RSoU}}(L)$ is polynomially related to the complexity of $L$ we obtain:

Corollary 9 The class of languages with bounded multi-party communication complexity contains languages with arbitrarily large time and space complexity.

In order to obtain also languages with essentially the largest possible circuit complexity we need codes that map $n$ bits into $O(n)$ bits. We can obtain such codes by concatenating Reed-Solomon codes with codes provided by the following lemma and the unary code $C_{U}$.

Lemma 10 For any integer $n \geq 1$, there exists a linear map $C_{8}:\{0,1\}^{n} \rightarrow G F[8]^{39 n}$ such that every $w \in C_{8}\left(\{0,1\}^{n}\right)$ is uniquely determined by any one-third of its coordinates.

By concatenating $C_{\mathrm{RS}}$ with $C_{8}$ and $C_{\mathrm{U}}$ we obtain the code $C_{\mathrm{RS} \text { (8) } \mathrm{U}}$ with polynomial time encoding and decoding that maps $n$ bit strings into $O(n)$ bit strings.

Corollary 11 For any $k \geq 3$, the class of languages with bounded $k$-party communication complexity contains languages with $2^{\Omega(n)}$ circuit complexity.

\section{Languages with a neutral letter}

A language $L \in \Sigma^{*}$ is said to have a neutral letter $e$ if for all $u, v \in \Sigma^{*}$ we have $u v \in L$ iff uev $\in L$. Thus, adding or deleting $e$ anywhere in a word $w$ does not affect membership in $L$. If a language has a neutral letter then membership in $L$ cannot depend, as in Lemma 7, on having specific value on a specific input position and, at least intuitively, this seems to take away a lot of the power inherent to the multiparty communication model. The neutral letter hypothesis was helpful in obtaining length lower bounds on bounded-width branching programs [4] and was central to the Crane-Beach Conjecture [3]. In this section, we give a precise characterization of languages with a neutral letter that have bounded $k$-party complexity for some fixed $k$. We first show that all such languages must be regular and then characterize them in terms of algebraic properties of their minimal automaton. 


\subsection{Proving Regularity}

Let $C \geq 0$ be an integer and let $\mathcal{G}$ be a family of functions over $\Sigma^{*}$ with finite range $R$. We say that inputs with weight at most $C$ determine the functions of $\mathcal{G}$ if every function $g: \Sigma \leq C \rightarrow R$ has at most one extension to $\Sigma^{*}$ in $\mathcal{G}$. Now, let $\mathcal{C}_{k, c}$ be the family of functions with a neutral letter and $k$-party communication complexity at most $c$. We show:

Lemma 12 There is a constant $C=C(k, c)$ such that functions of $\mathcal{C}_{k, c}$ are determined by inputs of weight at most $C$.

We obtain this lemma as a corollary to

Lemma 13 For any $C>0$ if the functions of $\mathcal{C}_{k, c}$ are not determined by inputs of size $C$ then Part ${ }_{k}$ can be solved by $k$ parties with $2 c+2$ communication for sets of size $C^{\prime}$ for some $C^{\prime} \geq C$.

Lemma 13 implies Lemma 12, since if there were no bound $C(k, c)$ as stated in Lemma 12, then Part $_{k}$ would have $k$-party communication complexity at most $2 c+2$ for arbitrary set size, resulting in a contradiction with Lemma 6.

Proof:(Lemma 13) For any word $w \in \Sigma^{*}$, we shall denote by $w_{e}$ the word obtained from $w$ by deleting all occurrences of $e$ in $w$. The ith letter of $w$ will be denoted by $w^{i}$. Also, for $k$ words $w_{1}, \ldots, w_{k}$, each of length $\ell$, let $w=w_{1} \diamond \ldots \diamond w_{k}$ denote the word obtained by interleaving the $k$ words in the following way : $|w|=\ell k$ and for all $1 \leq i \leq \ell k, w^{i}=w_{j}^{m}$ if $i=(m-1) k+j$ with $0<j<k+1$. Let us assume that $f$ and $g$ are in $\mathcal{C}_{k, c}$, such that they are not identical, but the minimal string $v \in\{\Sigma-e\}^{*}$ such that $f(v) \neq g(v)$ has length at least $C$. We consider the following $k$-party communication problem: each player gets $|v|$ bits on their forehead and let us denote the input on player $i$ 's forehead by $y_{i}$. (Note that from our comments following Lemma 6, the function Part $t_{k}$ requires unbounded $k$-wise complexity even if the input sets are known to be pairwise disjoint.) Consider a family of $k$ sets $I_{1}, \ldots, I_{k} \subseteq\{1, \ldots,|v|\}=[|v|]$, such that $I_{i} \cap I_{j}=\emptyset$ for all $i \neq j$. For each such choice of $k$ sets, we assign foreheads of the players in the following way: $y_{i}^{j}=v^{j}$ if $j \in I_{i}$, otherwise $y_{i}^{j}=e$. We define the function $h\left(y_{1}, \ldots, y_{k}\right)=1$ iff the corresponding family of $k$ subsets partitions $[|v|]$, i.e., $\cup_{i=1}^{k} I_{i}=[|v|]$. Notice that $h$ is exactly the partition problem for a basis set of size $|v| \geq C$. The reduction $\left(I_{1}, \ldots, I_{k}\right) \rightarrow\left(y_{1}, \ldots, y_{k}\right)$ is a rectangular reduction. We claim that $h\left(y_{1}, \ldots, y_{k}\right)=1$ iff $f\left(y_{1} \diamond \ldots \diamond y_{k}\right) \neq g\left(y_{1} \diamond \ldots \diamond y_{k}\right)$.

To see this we use the minimality property of $v$ : on words of length less than $|v| f$ and $g$ agree. For $y=y_{1} \diamond \ldots \diamond y_{k}$ we have $\left|y_{e}\right|=|v|$ only if $\cup_{i=1}^{k} I_{i}=[|v|]$ and in that case $y_{e}=v$ and $f(y) \neq g(y)$. Otherwise, we have $\left|y_{e}\right|<|v|$ and therefore $f(y)=g(y)$.

The function $f(v) \neq g(v)$ can be computed with $2 c$ bits of communication by running the $c$ bit protocol on $f$ and $g$ separately. For Part ${ }_{k}$ we also need to verify using two extra bits of communication that no row contains two ones. 
Let $f: \Sigma^{*} \rightarrow R$ be a function in $\mathcal{C}_{k, c}$ : For a word $w \in \Sigma^{*}$, we define the function $f_{w}: \Sigma^{*} \rightarrow R$ by $f_{w}(z)=f(w z)$. All the $f_{w}$ are also in $\mathcal{C}_{k, c}$ and so the functions $\left\{f_{w}\right\}$ are determined by inputs of length at most $C$. It follows that the equivalence relation on $\Sigma^{*}$ defined by $u \sim v$ iff $f(u z)=f(v z)$ for all $z \in \Sigma^{*}$ has at most $(|\Sigma|+1)^{C}$ equivalence classes. It is well-known that if $\sim$ has finite index then $f$ is regular and we obtain

Theorem 14 If $f$ is a function with a neutral letter such that $D_{k}(f)=O(1)$ for some fixed $k$, then $f$ is regular.

\subsection{Regular languages with bounded complexity}

A monoid $M$ is a set with a binary associative operation and a distinguished identity element $1_{M}$. A language $L \subseteq \Sigma^{*}$ is recognized by a finite monoid $M$ if there is a morphism $\phi$ from the free monoid $\Sigma^{*}$ to $M$ and a set $F \subseteq M$ such that $L=\phi^{-1}(F)$. A restatement of Kleene's Theorem states that $L$ is regular iff it is recognized by some finite monoid. If $L$ is regular, the syntactic monoid $M(L)$ of $L$ is the transformation monoid of $L$ 's minimal automaton [16] and is the smallest monoid recognizing $L$.

The word problem for $M$ is the function eval which maps a string $w=w_{1} \ldots w_{n} \in M^{*}$ to the product $\operatorname{eval}\left(w_{1} \ldots w_{n}\right)=w_{1} \cdot w_{2} \cdots w_{n}$. We define the $k$-party communication complexity of $M$, denoted $D_{k}(M)$ as the communication complexity of its word problem. Two of the authors gave a complete classification result for the two-party communication complexity of finite monoids [21] and this led to a similar classification for the two-party complexity of regular languages. The communication complexity of monoids was first studied in [18] from which we use the following:

Lemma 15 Let $L$ be a regular language with a neutral letter and let $M=M(L)$ be its syntactic monoid. Then for any $k \geq 2$ we have $D_{k}(L)=\Theta\left(D_{k}(M)\right)$.

A finite group is nilpotent if it is the direct product of $p$-groups and a monoid lies in the class $\overline{\mathbf{G}_{\text {nil }}}$ if all its subgroups are nilpotent. The class DO consists of monoids satisfying the identity $(x y)^{\omega}(y x)^{\omega}(x y)^{\omega}=(x y)^{\omega}$.

Lemma 16 If $M$ is a finite monoid outside of $\mathbf{D O}$ then $D_{k}(M)=\omega(1)$ for all $k$.

The lemma is proved in the appendix: we show that if $M$ lies outside DO then for any $k$ there exists a rectangular reduction of linear length from either $G I P_{k, p}$ or $\operatorname{Part}_{k}$ to the word problem of $M$.

Theorem 17 ([18]) Let $G$ be a group. If $G$ is in $\mathbf{G}_{\mathbf{n i l}}$ then there exists a constant $k \geq 2$ such that $D_{k}(G)=O(1)$. Otherwise $D_{k}(G)=\Omega(n)$ for all $k$.

In this case also, the lower bound is obtained through a rectangular reduction from $G I P_{k, p}$ to the word problem of any non-nilpotent finite group. The upper bound, on the other hand, stems 
from a combinatorial description of languages recognized by nilpotent groups. We say that a word $u=a_{1} \ldots a_{t}$ with $a_{i} \in \Sigma$ is a subword of the word $w$ if $w$ can be factorized as $w_{0} a_{1} w_{1} \ldots w_{t-1} a_{t} w_{t}$ and we denote by $\left(\begin{array}{l}w \\ u\end{array}\right)$ the number of such factorizations. We say that a language $L$ counts subwords of length $k$ modulo $m$ if membership of $w$ in $L$ depends on the values modulo $m$ of $\left(\begin{array}{c}w \\ u_{1}\end{array}\right), \ldots,\left(\begin{array}{l}w \\ u_{t}\end{array}\right)$ for some $u_{i}$ with $\left|u_{i}\right| \leq k$. One can show that the syntactic monoid of a regular language $L$ is a nilpotent group iff there exist $k, m \geq 2$ such that $L$ counts subwords of length $k$ modulo $m$ [23].

For $a \in \Sigma$ and $L, K \subseteq \Sigma^{*}$, the concatenation $L a K$ is said to be perfectly unambiguous if $L \subseteq$ $(\Sigma-\{a\})^{*}$ or $K \subseteq(\Sigma-\{a\})^{*}$. If $L a K$ is perfectly unambiguous then any $w \in L a K$ can be uniquely factorized as $w_{L} a w_{K}$ with $w_{L} \in L$ and $w_{K} \in K$ since the $a$ can only be the first or last occurrence of $a$ in $w$. Let $\mathcal{V}_{\Sigma}$ be the smallest class of regular languages over $\Sigma$ that contains both the subword-counting languages and the languages $\Sigma_{0}^{*}$ for each $\Sigma_{0} \subseteq \Sigma$ and which is closed under Boolean operations and perfectly unambiguous concatenations. The next lemma can be inferred from [21].

Lemma 18 A language $L \subseteq \Sigma^{*}$ is recognized by a monoid in $\mathbf{D O} \cap \overline{\mathbf{G}_{\mathbf{n i l}}}$ iff it is in $\mathcal{V}_{\Sigma}$.

We can now give a characterization of monoids that have bounded multiparty communication complexity for some suitably large constant $k$.

Theorem 19 Let $L \subseteq \Sigma^{*}$ be a regular language with a neutral letter and syntactic monoid $M$. If $M$ lies in $\mathbf{D O} \cap \overline{\mathbf{G}_{\text {nil }}}$ then there exists a constant $k$ such that $D_{k}(L)=O(1)$. Otherwise, we have $D_{k}(L)=\omega(1)$ for all $k$.

Proof: To obtain the upper bound, it suffices to show, by Lemma 18, that every language in $\mathcal{V}_{\Sigma}$ has bounded $k$-party complexity for some $k$ and we argue from the definition of $\mathcal{V}_{\Sigma}$.

First, any language $\Sigma_{0}^{*}$ has bounded two-party communication complexity since players only need to check that the input letters they have access to indeed belong to $\Sigma_{0}$. Furthermore, if $K$ counts subwords of length $k$ modulo $m$, then $D_{k+1}(K)=O(1)$ because any $k$-tuple of input letters is available to at least one player in the $(k+1)$-party game and the value of $\left(\begin{array}{l}w \\ u\end{array}\right)$ modulo $m$ can thus be computed with communication $k \cdot\lceil\log m\rceil$ if $|u| \leq k$. Clearly, Boolean combinations of languages with bounded $k$-party complexity also have bounded $k$-party complexity and it remains to show that if $L$ and $K$ have bounded $k$-party complexity and $L \subseteq(\Sigma-\{a\})^{*}$ then $L a K$ has bounded $(k+1)$-party complexity. Players proceed as follows: each party broadcasts the identity of the player which, in their opinion, holds on the forehead the first occurrence of $a$ in the input. This requires $k \cdot\lceil\log k\rceil$ bits of communication and the player holding that first occurrence will be the only dissenting voice since that letter is seen by all other parties. Since $k+1 \geq 3$, the $k$ remaining players now know the position of the first $a$ and they simulate the $k$-party protocols for $L$ and $K$ on the prefix and suffix at constant cost.

For the lower bound, if $M$ is not in DO then $D_{k}(M)=\omega(1)$ for all $k$ by Lemma 16. If $M$ contains a non-nilpotent group $G$ then $D_{k}(G)=\Omega(n)$ for all $k$ by Theorem 17 and we clearly have $D_{k}(M) \geq D_{k}(G)$. So for all $k$, we have $D_{k}(M)=\omega(1)$ and, by Lemma $15, D_{k}(L)=\omega(1)$.

Combining this result with Theorem 14 we get 
Theorem 20 If $L$ is a language with a neutral letter and bounded $k$-party communication complexity for some fixed $k$ then $L$ is regular and $M(L) \in \mathbf{D O} \cap \overline{\mathbf{G}_{\text {nil }}}$.

Note that the class $\mathbf{D O} \cap \overline{\mathbf{G}_{\text {nil }}}$ is decidable. Also, the corresponding regular languages have a nice logical characterization [22] and one can see from the definition of $\mathcal{V}_{\Sigma}$ that they all lie in $A C C^{0}$.

\section{Symmetric Functions}

For $w \in \Sigma^{*}$, we denote as $|w|_{a}$ the number of occurrences of $a$ in $w$. A function $f: \Sigma^{*} \rightarrow\{0,1\}$ is symmetric if its value depends only on the values $|w|_{a}$ for $a \in \Sigma$. Intuitively $k \geq 3$ parties computing a symmetric function only get limited benefits from the features of the multiparty model since their protocol cannot significantly rely on the precise set of input positions accessible to each player or on the fact that any $(k-1)$-tuple of bits is seen by one party. We formalize this idea by showing that any symmetric $f$ with bounded $k$-party complexity for a fixed $k$ in fact has bounded two-party complexity.

Let us first deal with functions with boolean inputs. To any symmetric function $f:\{0,1\}^{n} \rightarrow$ $\{0,1\}$ we naturally associate the function $\widehat{f}:\{0, \ldots, n\} \rightarrow\{0,1\}$ such that $f(x)=\widehat{f}\left(|x|_{1}\right)$ for every $x \in\{0,1\}^{n}$ and say that $f$ is $(\ell, r, p)$-periodic if $\widehat{f}(a)=\widehat{f}(a+p)$ for $\ell \leq a \leq n-r$.

Theorem 21 If $f:\{0,1\}^{n} \rightarrow\{0,1\}$ is symmetric and has bounded $k$-party communication complexity then in fact $f$ has bounded two-party complexity.

In the appendix, we extend this theorem to symmetric functions with non-Boolean domains. The result in the Boolean case is established through the next lemma. Recall that a simultaneous protocol is one in which each player sends a single message to an extra party (the referee) who then computes the answer solely based on the messages he received. In particular, the message sent by a party does not depend on messages sent by other parties. Since a $k$-party protocol of communication cost $c$ can be easily turned into a $k$-party simultaneous protocol with cost $c k 2^{c}$, functions of bounded complexity in the simultaneous model are exactly those with bounded complexity in the standard model.

Lemma 22 For any constants $k, c$ with $k \geq 1$ there exists an integer $N_{k+1}=N(k+1, c)$ such that every symmetric boolean function $f:\{0,1\}^{n} \rightarrow\{0,1\}$ that has a $k+1$-party simultaneous protocol of complexity c for the input partition in which players $X_{1}, \ldots, X_{k}$ each get $N_{k+1}$ bits and player $X_{k+1}$ gets the remaining $n-k N_{k+1}$ bits is $(\ell, r, p)$-periodic for some $\ell, r \leq k N_{k+1}$ and some $p \leq N_{k+1}$.

Theorem 21 then follows by observing that an $(\ell, r, p)$-periodic function has 2-party simultaneous communication complexity roughly $2 \cdot\lceil\log (\ell+r+p)\rceil$. The proof of Lemma 22 , given in the appendix, proceeds by induction on $k$. The base case is due to [20] and our induction step uses a nontrivial "player elimination" technique similar to that of [17]. More precisely, we use the Ramsey-like theorem of Gallai [10] to show that if $f$ has a $(k+1)$-party protocol of bounded cost then there exists a large set of inputs $\mathcal{P}$ for the foreheads of the first $k$ players on which player $P_{k+1}$ always sends the same communication. This renders the $(k+1)$ st player irrelevant if the input lies in $\mathcal{P}$ and allows the use of the induction hypothesis. 


\section{References}

[1] A. Ambainis. Upper bounds on multiparty communication complexity of shifts. In Proc. $13^{\text {th }}$ Symp. on Theoretical Aspects of Comp. Sci., pages 631-642, 1996.

[2] L. Babai, N. Nisan, and M. Szegedy. Multiparty protocols, pseudorandom generators for logspace, and time-space trade-offs. J. Comput. Syst. Sci., 45(2):204-232, 1992.

[3] D. A. M. Barrington, N. Immerman, C. Lautemann, N. Schweikardt, and D. Thérien. The Crane Beach conjecture. In Proc. 16th Symp. on Logic in Comp. Sci. (LICS-01), pages 187-196, 2001.

[4] D. A. M. Barrington and H. Straubing. Superlinear lower bounds for bounded-width branching programs. J. Comput. Syst. Sci., 50(3):374-381, 1995.

[5] P. Beame, T. Pitassi, and N. Segerlind. Lower bounds for Lovász-Schrijver systems and beyond follow from multiparty communication complexity. In Proc. 32nd Int. Conf. on Automata, Languages and Programming (ICALP'05), pages 1176-1188, 2005.

[6] P. Beame and E. Vee. Time-space tradeoffs multiparty communication complexity and nearest neighbor problems. In 34th Symp. on Theory of Computing (STOC'02), pages 688-697, 2002.

[7] A. K. Chandra, M. L. Furst, and R. J. Lipton. Multi-party protocols. In Proc. 15th ACM Symp. on Theory of Computing (STOC'83), pages 94-99, 1983.

[8] F. Chung and P. Tetali. Communication complexity and quasi-randomness. SIAM J. Discrete Math., 6(1):110-123, 1993.

[9] M. Goldmann and J. Håstad. Monotone circuits for connectivity have depth (log $)^{2-(1)}$. SIAM J. Comput., 27(5):1283-1294, 1998.

[10] R. L. Graham, B. L. Rotschild, and J. H. Spencer. Ramsey Theorey. Series in Discrete Mathematics. Wiley Interscience, 1980.

[11] V. Grolmusz. Separating the communication complexities of MOD $m$ and MOD $p$ circuits. In Proc. 33rd IEEE FOCS, pages 278-287, 1992.

[12] V. Grolmusz. The BNS lower bound for multi-party protocols in nearly optimal. Information and Computation, 112(1):51-54, 1994.

[13] V. Grolmusz. A weight-size trade-off for circuits and MOD $m$ gates. In Proc. $26^{\text {th }}$ ACM STOC, pages 68-74, 1994.

[14] E. Kushilevitz and N. Nisan. Communication Complexity. Cambridge University Press, 1997. 
[15] N. Nisan. The communication complexity of treshold gates. In Combinatorics, Paul Erdös is Eighty, Vol. 1, pages 301-315, 1993.

[16] J.-E. Pin. Syntactic semigroups. In Handbook of language theory, volume 1, chapter 10, pages 679-746. Springer Verlag, 1997.

[17] P. Pudlák. An application of Hindman's theorem to a problem on communication complexity. Combinatorics, Probability and Computing, 12(5-6):661-670, 2003.

[18] J.-F. Raymond, P. Tesson, and D. Thérien. An algebraic approach to communication complexity. Lecture Notes in Computer Science (ICALP'98), 1443:29-40, 1998.

[19] R. Raz. The BNS-Chung criterion for multi-party communication complexity. Computational Complexity, 9(2):113-122, 2000.

[20] M. Szegedy. Functions with bounded symmetric communication complexity, programs over commutative monoids, and ACC. J. Comput. Syst. Sci., 47(3):405-423, 1993.

[21] P. Tesson and D. Thérien. Complete classifications for the communication complexity of regular languages. Theory of Computing Systems, 38(2):135-159, 2005.

[22] P. Tesson and D. Thérien. Restricted two-variable sentences, circuits and communication complexity. In Proc. 32nd Int. Conf. on Automata, Languages and Programming (ICALP'05), pages 526-538, 2005.

[23] D. Thérien. Subword counting and nilpotent groups. In Combinatorics on Words: Progress and Perspectives, pages 195-208. Academic Press, 1983. 


\section{Appendix}

We give here proofs of lemmas that were omitted in the extended abstract.

\section{Proof of Lemma 10}

Lemma 10 For any integer $n \geq 1$, there exists a linear map $C_{8}:\{0,1\}^{n} \rightarrow G F[8]^{39 n}$ such that every $w \in C_{8}\left(\{0,1\}^{n}\right)$ is uniquely determined by any one-third of its coordinates.

Proof:

To prove the existence of our code we only need to prove the following claim.

Claim For $c \geq 37$, with high probability a random matrix over $G F[8]$ of dimension $n \times c n$ has the property that each submatrix of dimension $n \times c n / 3$ has rank $n$.

For any $n^{\prime}<n, n^{\prime}$ vectors over $G F[8]$ of length $c n / 3$ span less than $8^{n}$ different vectors. Thus the probability that a new random vector of length $\mathrm{cn} / 3$ falls into the space spanned by these vectors is at most $8^{n-c n / 3}$. Hence, the probability that a random matrix over $G F[8]$ of dimension $n$ by $c n / 3$ is of rank less than $n$ is at most $n \cdot 8^{n-c n / 3}$. (We pick the vectors step by step and at each step we fail to pick a linearly independent vector with probability at most $8^{n-c n / 3}$.) Thus the expected number of singular $n$ by $c n / 3$ submatrices of a random matrix of dimension $n$ by $c n$ is at most $n \cdot 8^{n-c n / 3} \cdot\left(\begin{array}{c}c n \\ c n / 3\end{array}\right)$. Since $\left(\begin{array}{c}c n \\ c n / 3\end{array}\right) \leq 2^{\mathrm{H}(1 / 3) c n}$, if $c \geq 37$ then $3-c+\mathrm{H}(1 / 3) c<0$ and the expected number of singular submatrices is $2^{-\epsilon n}$ for some $\epsilon>0$. The claim follows.

By concatenating $C_{\mathrm{RS}}$ with $C_{8}$ and $C_{\mathrm{U}}$ we obtain the code $C_{\mathrm{RS}} 8 \circ \mathrm{U}$ with polynomial time encoding and decoding that maps $n$ bit strings into $O(n)$ bit strings. Note that $C_{8}$ can be constructed by brute force in polynomial time as it is used only for strings of logarithmic length. Further speed-up can be achieved by using $C_{\mathrm{RS}} \mathrm{RS}$ 80 $\mathrm{U}$ codes where one would only need to construct $C_{8}$ for strings of log-log length. Using Fast Fourier Transform, $C_{\mathrm{RS}} \mathrm{RS} \circ 8 \circ \mathrm{U}$ can be encoded and decoded in time close to linear, whereby we obtain Corollary 11 .

\section{Proof of Lemma 16}

We want to establish

Lemma 16 If $M$ is a finite monoid outside of DO then $D_{k}(M)=\omega(1)$ for all $k$.

Recall from Section 4 that $\mathbf{D O}$ is the class of finite monoids satisfying $(x y)^{\omega}(y x)^{\omega}(x y)^{\omega}=(x y)^{\omega}$ for some $\omega \geq 1$. The following lemma (see e.g. [21]) gives a more useful characterization of DO. An element $e \in M$ is idempotent if $e=e^{2}$. 
Lemma 23 If the finite monoid $M$ is not in DO then either

1. There exist idempotents $a, b \in M$ and an integer $p \geq 2$ such that $(a b a)^{p}=a$ but $(a b a)^{t} \neq a$ if $1 \leq t \leq p-1$

2. There exist elements $a, b \in M \times M$ such that ab is idempotent but for all $x, y \in M \times M$ we have $x a^{2} y \neq a b$ and $x b^{2} y \neq a b$.

We can now proceed to establish Lemma 16.

Proof: (Lemma 16)

Suppose first that there are idempotents $a, b \in M$ such that $(a b a)^{p}=a$ but $(a b a)^{t} \neq a$ if $1 \leq t \leq$ $p-1$. We claim that for any $k$ there is a linear-length rectangular reduction from $G I P_{k, p}$ to the word problem of $M$. The reduction maps an $n \times k$ instance $A$ of $G I P_{k, p}$ to a string of $(k+2) n$ elements of $M$ with each block of $k+2$ elements corresponding to a column of $A$. The first and last elements of each block are always $a$ and the $(i+1)$ th element of the block is a $b$ if the $i$ th bit of the column is 0 and the identity $1_{M}$ otherwise. Since $b$ is idempotent, the output of each such block thus multiplies out to $a b a$ if some bit in the column is 0 and to $a$ otherwise. Hence, the value of the whole product is $a$ iff the number of all 1 columns is 0 modulo $p$. Since $D_{k}\left(G I P_{k, p}\right)=\Omega(n)$, we have $D_{K}(M)=\Omega(n)$ because the length of the reduction is linear.

Suppose that there are elements $a, b \in M \times M$ such that $a b$ is idempotent but for all $s, t \in M \times M$ we have $s a^{2} t \neq a b$ and $s b^{2} t \neq a b$. Then we claim that $\operatorname{Part}_{k}$ reduces to the word problem of $M \times M$. Again, our reduction produces $n$ blocks of $k+2$ elements of $M \times M$. The first element of each block is always an $a$ and the last one is always $a b$, while the $(i+1)$ th element is $b$ if the $i$ th bit of the column is 1 and the identity $1_{M \times M}$ otherwise. Thus, if a column of $A$ contains $r$ 's, the product of monoid elements in the corresponding block is $a b^{r} a b$. The product of the $n$ blocks is thus $(a b)^{2 n}=a b$ if each column contains exactly one 1 . If some column of $A$ contains two or more 1's, then the corresponding block evaluates to $a\left(b^{2}\right) b^{r-2} a b$ and so the product of the $n$ blocks can be written as $x b^{2} y$ and cannot be $a b$. Similarly, if a column is all 0 , the corresponding block evaluates to $a a b$ and the $n$ blocks multiply out to some $x a^{2} y \neq a b$. Since $D_{k}\left(\operatorname{Part}_{k}\right)=\omega(1)$, we get $D_{k}(M \times M)=\omega(1)$. Furthermore $D_{k}(M \times M)$ is at most $2 \cdot D_{k}(M)$ so we also get $D_{k}(M)=\omega(1)$.

\section{Proof of Lemma 22}

We now prove:

Lemma 22 For any constants $k, c$ with $k \geq 1$ there exists an integer $N_{k+1}=N(k+1, c)$ such that every symmetric boolean function $f:\{0,1\}^{n} \rightarrow\{0,1\}$ that has a $k+1$-party simultaneous protocol of complexity c for the input partition in which players $X_{1}, \ldots, X_{k}$ each get $N_{k+1}$ bits and player $X_{k+1}$ gets the remaining $n-k N_{k+1}$ bits is $(\ell, r, p)$-periodic for some $\ell, r \leq k N_{k+1}$ and some $p \leq N_{k+1}$. 
We recall definitions and notations of Section 5: to any symmetric function $f:\{0,1\}^{n} \rightarrow\{0,1\}$ we naturally associate the function $\widehat{f}:\{0, \ldots, n\} \rightarrow\{0,1\}$ such that $f(x)=\widehat{f}\left(|x|_{1}\right)$ for every $x \in\{0,1\}^{n}$. A symmetric boolean function $f$ on $n$ variables is $(\ell, r, p)$-periodic if $\widehat{f}(a)=\widehat{f}(a+p)$ for $\ell \leq a \leq n-r$.

In order to specify $N(k, c)$ we need to recall the following Ramsey-type theorem which will be used in our proof. This theorem is a consequence of Gallai's Theorem (see page 38 and the example on page 39 in [10]).

Proposition 24 For any integers $C, k, m>0$, there is an integer $R=R(C, k, m)$ such that for each $C$-coloring of $\{0, \ldots, R\}^{k}$, there exist $x_{1}^{0}, \ldots, x_{k}^{0}<R$ and $1 \leq d<R$ such that all points of the set $\mathcal{P}=\left\{\left(x_{1}, \ldots, x_{k}\right): x_{i}=x_{i}^{0}+d y_{i}, 0 \leq y_{i} \leq m\right\}$ have the same color and all lie in $\{0, \ldots, R\}^{k}$.

Now, $N(k+1, c)$ is defined by induction on $k$. We set $N(2, c)=2^{c}+1$ and for $k \geq 2, N(k+$ $1, c)=R\left(2^{c}, k, N(k, c) !+2(k-1) N(k, c)\right)$. We are ready to prove lemma 22.

Proof: (Lemma 22) Our idea is the following: given a constant cost $(k+1)$-party protocol for the symmetric function $f$, we use the Ramsey-theoretic fact to 'eliminate' the $(k+1)$ st player by restricting $f$ to a set of inputs on which that player's message is always the same. This enables us to construct a bounded cost $k$-party symmetric function $f^{\prime}$ closely related to $f$. Our inductive hypothesis applies to $f^{\prime}$ and we show that the periodicity of $f^{\prime}$ implies the periodicity of $f$.

For $k=1$ the lemma was observed in [20] and we briefly mention the argument here. By the pigeonhole principle, there are two inputs $x$ and $x^{\prime}$ for the first player with $|x|_{1}<\left|x^{\prime}\right|_{1}<2^{c}+1=$ $N(2, c)$ such that the second player sends the same message when $x$ or $x^{\prime}$ are on the first player's forehead. One can verify that $f$ is $\left(|x|_{1},\left|x^{\prime}\right|_{1}-|x|_{1},\left|x^{\prime}\right|_{1}-|x|_{1}\right)$-periodic: since the referee does not know whether the second player sees $x$ or $x^{\prime}$ we must have $\widehat{f}(y)=\widehat{f}\left(y+\left|x^{\prime}\right|_{1}-|x|_{1}\right)$ for any $|x|_{1} \leq y \leq n-|x|_{1}+\left|x^{\prime}\right|_{1}$. We now prove the lemma by induction on $k$ for $k \geq 2$.

Let $\Pi$ be a simultaneous $(k+1)$-player protocol of cost $c$ that computes $f$ under a partition of the following form. Players $1, \ldots, k$ each have $N_{k+1}$ bits written on the forehead, and player $k+1$ gets the remaining $n-k N_{k+1}$ bits. Color each point $\left(x_{1}, \ldots, x_{k}\right) \in\left\{0, \ldots, N_{k+1}\right\}^{k}$ by the communication of the $(k+1)$-st player when $1^{x_{i}} 0^{N_{k+1}-x_{i}}$ is on the forehead of the player $i$. By Proposition 24 there is a set $\mathcal{P}$ of points in $\left\{0, \ldots, N_{k+1}\right\}^{k}$, such that player $k+1$ sends the same message for every point in

$$
\mathcal{P}=\left\{\left(x_{1}, \ldots, x_{k}\right): x_{i}=x_{i}^{0}+d y_{i}, 0 \leq y_{i} \leq N_{k} !+2(k-1) N_{k}\right\},
$$

for some $1 \leq d<N_{k+1}$ and some $x_{i}^{0}<N_{k+1}$.

Let $\ell=d(k-1) N_{k}+\sum_{i=1}^{k} x_{i}^{0}, r=k N_{k+1}$, and $p=d \cdot N_{k}$ !. Clearly, $\ell, r, p$ satisfy the required bounds from the lemma. Let $x \in\{\ell, \ell+1, \ldots, n-r\}$. We claim that $\widehat{f}(x)=\widehat{f}(x+p)$.

Define a function $\widehat{f}_{x}^{\prime}:\left\{0, \ldots, N_{k} !+2(k-1) N_{k}\right\} \rightarrow\{0,1\}$ by setting

$$
\widehat{f}_{x}^{\prime}(u)=\widehat{f}\left(x+u d-d(k-1) N_{k}\right)
$$


The function $\widehat{f}_{x}^{\prime}$ corresponds to a symmetric function $f_{x}^{\prime}:\{0,1\}^{N_{k} !+2(k-1) N_{k}} \rightarrow\{0,1\}$. We claim that there is a $c$ bit $k$-party communication protocol for $f_{x}^{\prime}$ under the input partition in which the first $k-1$ players get $N_{k}$ bits on their foreheads and the remaining bits are on player $k$ 's forehead. Indeed, suppose that the players 1 through $k$ have on their foreheads strings of weights $y_{1}$ through $y_{k}$. To compute $\widehat{f}_{x}^{\prime}\left(\sum_{i=1}^{k} y_{i}\right)$ the players take advantage of the fact that

$$
\widehat{f}_{x}^{\prime}\left(\sum_{i=1}^{k} y_{i}\right)=\widehat{f}\left(x+\left(\sum_{i=1}^{k} y_{i} d\right)-d(k-1) N_{k}\right)=\widehat{f}\left(\left(\sum_{i=1}^{k} x_{i}^{0}+y_{i} d\right)+\left(x-d(k-1) N_{k}-\sum_{i=1}^{k} x_{i}^{0}\right)\right) .
$$

Consequently, the players simply simulate the protocol $\Pi$ (which computes $f$ ) on the input where players $1, \ldots, k$ have on their respective forehead strings of weight $x_{1}^{0}+d y_{1}, x_{2}^{0}+d y_{2}, \ldots, x_{k}^{0}+d y_{k}$ and the player $k+1$ has on its forehead a string of weight $x-d(k-1) N_{k}-\sum_{i=1}^{k} x_{i}^{0}$. The simulation of the missing party poses no problem since the inputs of the first $k$ players belong to $\mathcal{P}$.

By the induction hypothesis, $f_{x}^{\prime}$ is periodic for some $\ell^{\prime}, r^{\prime} \leq(k-1) N_{k}$ and $p^{\prime}<N_{k}$. Hence, $\widehat{f}(x)=\widehat{f}_{x}^{\prime}\left((k-1) N_{k}\right)=\widehat{f}_{x}^{\prime}\left(N_{k} !+(k-1) N_{k}\right)=\widehat{f}\left(x+d N_{k} !\right)$, where we use the facts that $p^{\prime}$ divides $N_{k} !,(k-1) N_{k} \geq \ell^{\prime}$ and $N_{k} !+(k-1) N_{k} \leq N_{k} !+2(k-1) N_{k}-r^{\prime}$. This concludes the proof.

Theorem 21 now follows as well as:

Corollary 25 If $f: \Sigma^{n} \rightarrow\{0,1\}$ is symmetric and has bounded $k$-party communication complexity then in fact $f$ has bounded two-party complexity.

Proof: Let $\Sigma=\left\{a_{1}, \ldots, a_{t}\right\}$. For any $\Sigma^{0} \subseteq \Sigma$ and any word $w$ in $\left(\Sigma-\Sigma^{0}\right)^{*}$, we denote as $f_{w}^{\Sigma_{0}}$ the symmetric function over alphabet $\Sigma^{0}$ defined by $f_{w}^{\Sigma_{0}}(x)=f(w x)$. We now argue by induction on $t$ the cardinality of $\Sigma$. Our base case is Theorem 21. If $t \geq 3$ then let $\Sigma_{0}=\left\{a_{1}, a_{2}\right\}$ Since $f$ has bounded $k$-party complexity then so does $f_{w}^{\Sigma^{0}}$ for any $w$. Applying our result for binary alphabets we get that for any $w$ we get that $f_{w}^{1,2}$ is $(t, r, \rho)$-periodic for $t=r=(k-1) N_{k}$ and $\rho=N_{k}$ !. In particular this means that the function $f_{x}^{\Sigma-\Sigma_{0}}$ is determined by the numbers $|x|_{a_{1}}$ and $|x|_{a_{2}}$ up to the thresholds $t, r$ and modulo $\rho$. This can be computed at constant cost by two players and since $f_{x}^{\Sigma-\Sigma_{0}}$ is a symmetric with bounded $k$-party communication complexity over an alphabet of cardinality smaller than $t$ it can be evaluated at constant bounded two-party cost by our induction hypothesis. 